Article

\title{
Evaluation of the CRISPR/Cas9 Genetic Constructs in Efficient Disruption of Porcine Genes for Xenotransplantation Purposes Along with an Assessment of the Off-Target Mutation Formation
}

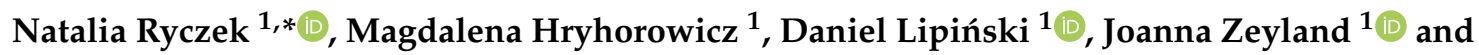 \\ Ryszard Słomski ${ }^{2}$ \\ 1 Department of Biochemistry and Biotechnology, Poznan University of Life Sciences, Dojazd 11, \\ 60-632 Poznań, Poland; magdalena.hryhorowicz@gmail.com (M.H.); lipinskidaniel71@gmail.com (D.L.); \\ jzeyland@gmail.com (J.Z.) \\ 2 Institute of Human Genetics, Polish Academy of Sciences, Strzeszyńska 32, 60-479 Poznań, Poland; \\ ryszard.slomski@up.poznan.pl \\ * Correspondence: nataliaryczek.nr@gmail.com
}

Received: 21 May 2020; Accepted: 24 June 2020; Published: 26 June 2020

\begin{abstract}
The increasing life expectancy of humans has led to an increase in the number of patients with chronic diseases and organ failure. However, the imbalance between the supply and the demand for human organs is a serious problem in modern transplantology. One of many solutions to overcome this problem is the use of xenotransplantation. The domestic pig (Sus scrofa domestica) is currently considered as the most suitable for human organ procurement. However, there are discrepancies between pigs and humans that lead to the creation of immunological barriers preventing the direct xenograft. The introduction of appropriate modifications to the pig genome to prevent xenograft rejection is crucial in xenotransplantation studies. In this study, porcine GGTA1, CMAH, $\beta 4$ GalNT2, vWF, ASGR1 genes were selected to introduce genetic modifications. The evaluation of three selected gRNAs within each gene was obtained, which enabled the selection of the best site for efficient introduction of changes. Modifications were examined after nucleofection of porcine primary kidney fibroblasts with CRISPR/Cas9 system genetic constructs, followed by the tracking of indels by decomposition (TIDE) analysis. In addition, off-target analysis was carried out for selected best gRNAs using the TIDE tool, which is new in the research conducted so far and shows the utility of this tool in these studies.
\end{abstract}

Keywords: xenoantigen; coagulation system dysregulation; CRISPR/Cas9 system; genome modifications; non-homologous DNA ends joining (NHEJ); TIDE analysis; off-target

\section{Introduction}

The increasing life expectancy of humans has led to an increase in the number of patients with chronic diseases and organ failure. Organ transplantation is an effective approach in the treatment of the end-stage organ failure. However, the imbalance between the supply and the demand for human organs is a serious problem in modern transplantology. In view of the above, it is assumed that alternative approaches would reduce or eliminate this problem. One of many solutions is the use of xenotransplantation [1,2].

Xenotransplantation is any procedure that involves the transplantation, implantation, or infusion of a recipient (in this case a human) with zoonotic cells, tissues, or organs. In addition, therapies using human body fluids, tissues, organs, or cells that have had ex vivo contact with animal organs, tissues, 
or cells are also subject to this term [3]. The domestic pig (S. s. domestica) is currently considered as the most suitable species for human organ procurement. The reasons for choosing the pig as a donor animal include its relatively large litter size and short puberty, the size and physiological similarity of its organs to human organs, and the low risk of xenozoonosis transmission [4]. However, there are discrepancies between pigs and humans that lead to the creation of immunological barriers preventing the direct xenograft. These differences cause xenograft rejection [5]. The introduction of appropriate modifications into the porcine genome to prevent xenograft rejection is crucial in xenotransplantation studies. There are three main types of xenograft rejection consecutively-hyperacute rejection (HAR), acute humoral xenograft rejection (AHXR), and acute cellular rejection (ACR) [6-8]. In addition, dysregulation of the recipient's coagulation system is a barrier in xenotransplantation, which appears in parallel with HAR, AXHR, and ACR $[9,10]$. Accordingly, this study focuses on preventing HAR and coagulation dysregulation in xenotransplantation.

HAR is a process that occurs within a few minutes to several hours after xenotransplantation. HAR is a type of humoral rejection mediated by IgM antibodies naturally occurring in the recipient. The association of recipient antibodies with epitopes present on the porcine endothelial cells activates the complement system [11]. To date, three epitopes have been described that constitute a barrier to xenotransplantation and are responsible for HAR. Galactose- $\alpha 1,3$-galactose $(\alpha-G a l)$ is the major xenoantigen involved in xenograft hyperacute rejection. This epitope is synthesized by $\alpha-1,3$-galactosyltransferase encoded by the porcine GGTA1 gene [11,12]. The second significant epitope is Neu5Gc, which is formed in an enzymatic reaction involving cytidine monophospho-N-acetylneuraminic acid hydroxylase encoded by the porcine CMAH gene $[13,14]$. $\beta-1,4 \mathrm{~N}$-acetylgalactosaminyltransferase 2 encoded by the porcine $\beta 4$ GalNT2 gene is involved in the synthesis of Sd(a) antigen [15-17].

Dysregulation of the recipient's coagulation system is one of the main barriers in xenotransplantation. It causes the development of thrombotic microangiopathy in xenograft. Features of thrombotic microangiopathy include fibrin deposition and platelet aggregation, which causes thrombosis within the transplant blood vessels and ultimately ischemic damage [18]. With the development of disorders of the coagulation system, systemic consumption coagulopathy is often observed in the recipient, which can lead to his death [19]. There are also factors expressed in specific organs that pose a problem in xenotransplantation. One of them is the von Willebrand factor encoded by the porcine $v W F$ gene, which is involved in the pathogenesis of transplant failure in lung xenotransplantation $[20,21]$. Fatal thrombocytopenia accompanying liver xenotransplantation is another barrier resulting from differences in the coagulation system. Human platelets are bound by asialoglycoprotein receptor (ASGR) encoded by porcine ASGR1 and ASGR2 genes. They are expressed in liver sinusoidal endothelial cells (LSEC) [22,23].

The described research focuses on introducing changes within genes that are involved in the immune response that is the biggest barrier in xenotransplantation. Porcine GGTA1, CMAH, and $\beta 4$ GalNT2 genes involved in the synthesis of epitopes responsible for the xenograft hyperacute rejection. Additionally, two genes responsible for the synthesis of porcine proteins causing dysregulation of the recipient's coagulation system have been chosen-the porcine $v W F$ gene and porcine ASGR1 gene.

The use of the CRISPR/Cas9 system for genome modification has led to enormous progress in the field of animal transgenesis [24]. Through projectable short gRNA, it is possible to precisely generate double-strand DNA breaks (DSBs). DSBs of DNA generated by the CRISPR/Cas9 system are repaired by NHEJ or by homologous directed repair (HDR) after delivery of properly designed donor DNA $[25,26]$. There are, however, some restrictions related to the use of the CRISPR/Cas9 system. One of them is off-target effect [27]. In this study, the usefulness of the TIDE online tool has been confirmed not only to analyze indel-type mutations arising as a result of DSBs repair via NHEJ but also to analyze additional Cas9 hydrolysis sites linked to a specific gRNA—off-target sites. 


\section{Materials and Methods}

\subsection{Selection of Short Oligonucleotides}

Short oligonucleotides (gRNA) and PCR primers were selected using the Benchling platform, San Francisco, CA, USA (www.benchling.com). Nucleotides have been added to the selected gRNA sequences at the $5^{\prime}$ and $3^{\prime}$ ends for molecular cloning. The designed oligonucleotides were ordered from the Institute of Biochemistry and Biophysics Polish Academy of Sciences.

\subsection{Preparation of Genetic Constructions in the CRISPR/Cas9 System}

The constructions were prepared using the vector $p S p C a s 9(B B)-2 A-P u r o(P X 459)$ V2.0, which was a gift from Feng Zhang (Addgene plasmid \#62988; http://n2t.net/addgene:62988; RRID: Addgene_62988). For each pair of oligonucleotides, a hybridization mixture containing $100 \mu \mathrm{M} F$ and R gRNA sequences was prepared. The mixtures were incubated $5 \mathrm{~min}$ at $95^{\circ} \mathrm{C}$ and then incubated at room temperature for 10 min. Plasmid DNA was hydrolyzed with the restriction enzyme BbsI-HF ${ }^{\circledR}$ (New England Biolabs, Ipswich, MA, USA) for $1.5 \mathrm{~h}$ at $37^{\circ} \mathrm{C}$. To combine oligonucleotides after hybridization with plasmids in linear form, ligation was performed using T4 DNA Ligase (New England Biolabs, Ipswich, MA, USA). The mixtures were incubated for $18 \mathrm{~h}$ at $16^{\circ} \mathrm{C}$, followed by additional hydrolysis with the use of enzyme BbsI-HF ${ }^{\circledR}$ for 30 min at $37^{\circ} \mathrm{C}$. Then Escherichia coli bacterial cells were transformed with purified constructions and positive clones were selected. Plasmid DNA isolation was performed using the Plasmid Maxi Kit (Qiagen, Germantown, MD, USA).

\subsection{Isolation and Culture of Porcine Primary Kidney Cells In Vitro}

For the isolation of porcine primary kidney fibroblasts, a $2 \times 2 \times 2 \mathrm{~cm}$ kidney cortical tissue was excised. Then, after tissue fragmentation, enzymatic-mechanical disintegration was carried out using collagenase II (Sigma-Aldrich, Darmstadt, Germany) and a magnetic stirrer. Then the incubation was carried out for $60 \mathrm{~min}$ while heating the mixture to $37^{\circ} \mathrm{C}$. The mixture was then filtered through a filter (0.5 mm pore diameter) and washed several times in the culture medium (DMEM (Sigma-Aldrich, Darmstadt, Germany), 20\% FBS (Sigma-Aldrich, Darmstadt, Germany), 1\% MEM Non-Essential Amino Acid Solution (100×) (Sigma-Aldrich, Darmstadt, Germany)), 1\% Antibiotic Antimycotic Solution $(100 \times)$ (Sigma-Aldrich, Darmstadt, Germany), 1\% Sodium pyruvate solution (100 mM) (Sigma-Aldrich, Darmstadt, Germany), 1\% L-Glutamine solution (200 mM) (Sigma-Aldrich, Darmstadt, Germany)). In vitro cell culture was carried out under standard aseptic conditions $\left(5 \% \mathrm{CO}_{2}, 37^{\circ} \mathrm{C}\right)$.

\subsection{Nucleofection}

Primary porcine kidney fibroblasts were detached from the surface of T-flasks by Accutase ${ }^{\circledR}$ solution (Sigma-Aldrich, Darmstadt, Germany) and counted using a Scepter ${ }^{\mathrm{TM}}$ Handheld Automated Cell Counter, version 2.0 (Merck, Darmstadt, Germany). One million cells were used for the transfection. Nucleofection was carried out using the Mouse Embryonic Fibroblast Nucleofector ${ }^{\mathrm{TM}}$ Kit 1 (Lonza, Basel, Switzerland) and program T-007 on Amaxa ${ }^{\mathrm{TM}}$ Nucleofector $^{\mathrm{TM}}$ II device (Lonza, Basel, Switzerland).

\subsection{Antibiotic Selection}

After $24 \mathrm{~h}$ since performing nucleofection, the culture medium has been changed to selective culture medium with the addition of puromycin at a concentration of $1 \mu \mathrm{g} / \mathrm{mL}$. Incubation was then carried out for $48 \mathrm{~h}$, after which the selection medium was removed, and standard medium was added again. The culture was carried out until the expected cell confluency was obtained.

\subsection{Analysis of Introduced Genetic Modifications}

Prior to analysis of the introduced genetic modifications, DNA isolation from modified primary porcine kidney fibroblasts was performed using the DNeasy Blood \& Tissue Kit (Qiagen, Germantown, 
MD, USA). Then PCR reactions were performed using the StartWarm HS-PCR Mix (A\&A Biotechnology, Gdynia, Poland) to amplify DNA fragments that include modified loci and DNA fragments containing potential off-target sites. The purified PCR products were sequenced in the Sequencing Laboratory of the Faculty of Biology at the Adam Mickiewicz University in Poznan.

\subsection{Sequencing Analysis Using the TIDE Tool}

The presence of modifications at the target locus and off-target sites after nucleofection of primary porcine kidney fibroblasts was identified using the TIDE online tool, version 2.0.1 by The Netherlands Cancer Institute, Amsterdam, Netherlands (https://tide.deskgen.com/).

\section{Results}

\subsection{Selection of the Potential Modifications Location in Porcine Genome}

The first stage of research was to find the best bioinformatically selected gRNA using the Benchling platform. The choice was made based on several guidelines: the localization of the introduced modifications, the values of the on-target score (from 0 to 100-the higher score, the better) and the off-targets score (from 0 to 100-the higher the score, the lower the chance of additional genomic cleavage sites). The localization of the three selected gRNAs for each of the tested loci in the porcine genome is shown in Table 1.

Table 1. Localization of the potential modification loci in porcine genome.

\begin{tabular}{|c|c|c|c|}
\hline Porcine Genome Locus & gRNA & Exon & Chromosome Localization $^{1}$ \\
\hline \multirow{3}{*}{ GGTA1 } & gGGTA1 F1/R1 & Exon 8 & Chromosome 1, c261513705-261513686 (NC_010443.5) \\
\hline & gGGTA1 F2/R2 & Exon 8 & Chromosome 1, c261513541-261513522 (NC_010443.5) \\
\hline & gGGTA1 F3/R3 & Exon 8 & Chromosome 1, c261513764-261513745 (NC_010443.5) \\
\hline \multirow{3}{*}{ CMAH } & gCMAH F1/R1 & Exon 6 & Chromosome 7, c19902027-19902008 (NC_010449.5) \\
\hline & gCMAH F2/R2 & Exon 3 & Chromosome 7, c19917616-19917597 (NC_010449.5) \\
\hline & gCMAH F3/R3 & Exon 5 & Chromosome 7, c19903792-19903773 (NC_010449.5) \\
\hline \multirow{3}{*}{$\beta 4$ GalNT2 } & gß4GalNT2 F1/R1 & Exon 2 & Chromosome 12, c25388178-25388159 (NC_010454.4) \\
\hline & $\mathrm{g} \beta 4 \mathrm{GalNT} 2 \mathrm{~F} 2 / \mathrm{R} 2$ & Exon 3 & Chromosome 12, c25386323-25386304 (NC_010454.4) \\
\hline & $\mathrm{g} \beta 4$ GalNT2 F3/R3 & Exon 6 & Chromosome 12, c25381330-25381311 (NC_010454.4) \\
\hline \multirow{3}{*}{$v W R$} & gvWR F1/R1 & Exon 2 & Chromosome 5, 64553818-64553837 (NC_010447.5) \\
\hline & gvWR F2/R2 & Exon 3 & Chromosome 5, 64556041-64556060 (NC_010447.5) \\
\hline & gvWR F3/R3 & Exon 4 & Chromosome 5, 64557621-64557640 (NC_010447.5) \\
\hline \multirow{3}{*}{ ASGR1 } & gASGR1 F1/R1 & Exon 3 & Chromosome 12, c52538530-52538511 (NC_010454.4) \\
\hline & gASGR1 F2/R2 & Exon 7 & Chromosome 12, c52537633-52537614 (NC_010454.4) \\
\hline & gASGR1 F3/R3 & Exon 9 & Chromosome 12, c52537146-52537127 (NC_010454.4) \\
\hline
\end{tabular}

${ }^{1}$ Based on NCBI: Sus scrofa isolate TJ Tabasco breed Duroc chromosome 12, Sscrofa11.1, whole genome shotgun sequence, GCF_000003025.6.

\subsection{Analysis of On-Target Modification Sites}

\subsubsection{Nucleofection Efficiency}

To obtain genetically modified primary porcine renal fibroblasts, nucleofections were performed. Transfection efficiency for isolated cells was checked using the pmaxGFP ${ }^{T M}$ Vector from the nucleofection kit and visualized using a ZOE Fluorescent Cell Imager (Figure 1). After counting 10,000 cells from multiple views, transfection efficiency was estimated to be around $65-70 \%$. 


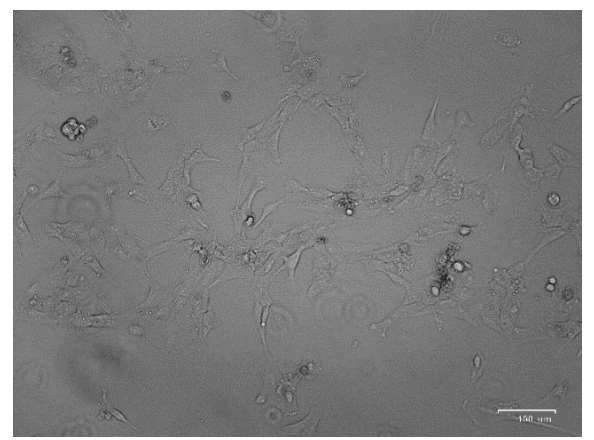

(a)

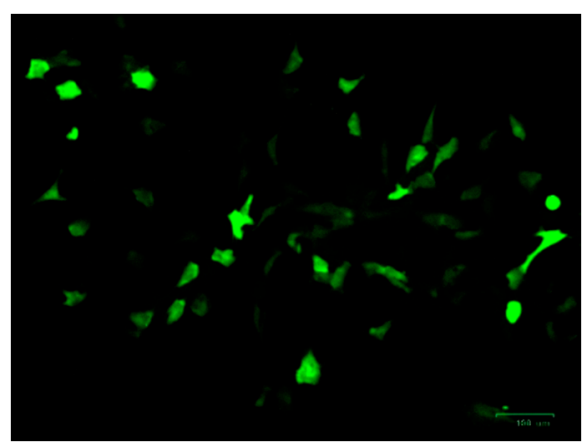

(b)

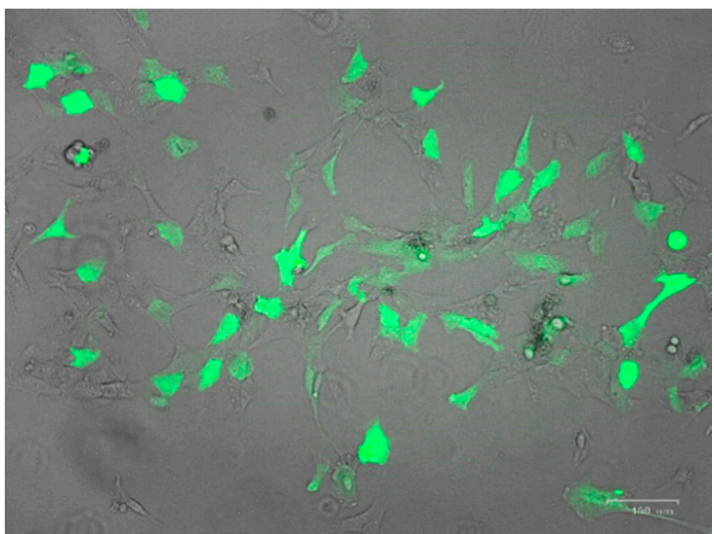

(c)

Figure 1. Nucleofection efficiency obtained on porcine primary kidney fibroblasts. Cells visualization was performed using a ZOE Fluorescent Cell Imager $24 \mathrm{~h}$ after nucleofection-(a) brightfield; (b) fluorescence detection lamp; (c) merge of view (a,b). The scale is $100 \mu \mathrm{m}$.

3.2.2. The Efficiency of Introducing Modifications within the Examined Porcine Genes Using Genetic Constructions Selected as the Best

PCR products obtained on DNA template isolated from modified and unmodified porcine primary kidney fibroblasts were purified using a CleanUp kit (A\&A Biotechnology, Gdynia, Poland). Then, sequencing was commissioned, the results of which were analyzed using the TIDE online tool, version 2.0.1. One, best gRNA from the three directed at each of the examined genes was selected.

The gGGTA1 F1/R1 was chosen as the best for the disruption of the porcine GGTA1 gene. The gGGTA1 F1/R1, when combined with Cas9, enabled on modification of porcine primary kidney fibroblasts with total efficiency of $70.1 \%$. A statistically significant mutation occurring with the highest frequency was a single nucleotide deletion in $51.1 \%$ sequences. It was determined that the insertion of one nucleotide occurred at $11.6 \%$ (adenosine nucleotide was incorporated in $47.6 \%$ sequences).

From the genetic constructs of the CRISPR/Cas9 system directed to the porcine CMAH gene, the one containing gCMAH F3/R3 was chosen as the best, as it enabled obtaining $61.2 \%$ total efficiency of modification. The most common mutation was the insertion of a single nucleotide, occurring in $57 \%$ of the tested sequences. In $93.6 \%$ of sequences, the inserted nucleotide was the cytidine.

The next gene tested was porcine $\beta 4 G$ alNT2. For genetic construction containing g $\beta 4$ GalNT2 F3/R3, a total modification efficiency of $45.2 \%$ was obtained. The most common mutation was a deletion of one nucleotide, which occurred in $28.8 \%$ sequences. The second most common mutation was the insertion of a single nucleotide, occurring in $8.4 \%$ of cases. Adenosine nucleotide was inserted in $79.8 \%$ of sequences. 
Another analysis was performed for genetic constructions directed at the porcine $v W F$ gene. A total efficiency of $85.1 \%$ was obtained for the plasmid containing gvWF F2/R2. The most common deletion of one nucleotide occurred with a frequency of $39.9 \%$. The second most common mutation was the insertion of one nucleotide, which occurred in $34.3 \%$ of sequences. An adenosine nucleotide was inserted in $83.6 \%$ of sequences.

The last gene tested was porcine ASGR1. A total efficiency of $80.5 \%$ was obtained for the plasmid containing gASGR1 F3/R3. The most common deletion of one nucleotide occurred in $58.6 \%$ of sequences. The second most common mutation was the deletion of two nucleotides.

Detailed results of indel spectrum and inserted nucleotide probability for all chosen as the best gRNAs are presented in Figure 2.
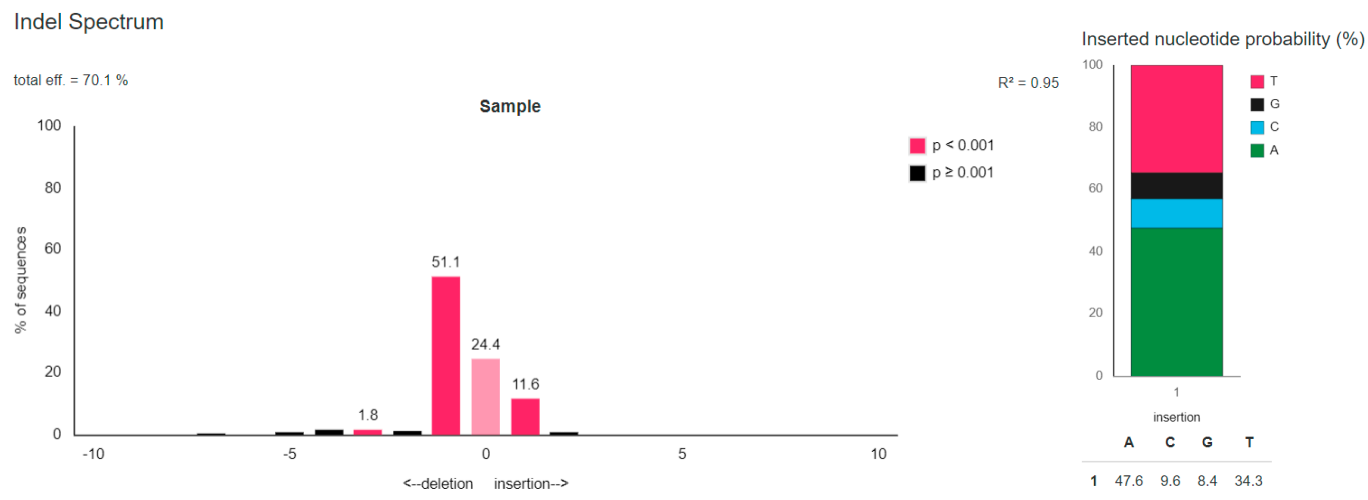

(a) gGGTA1 F1/R1

Indel Spectrum
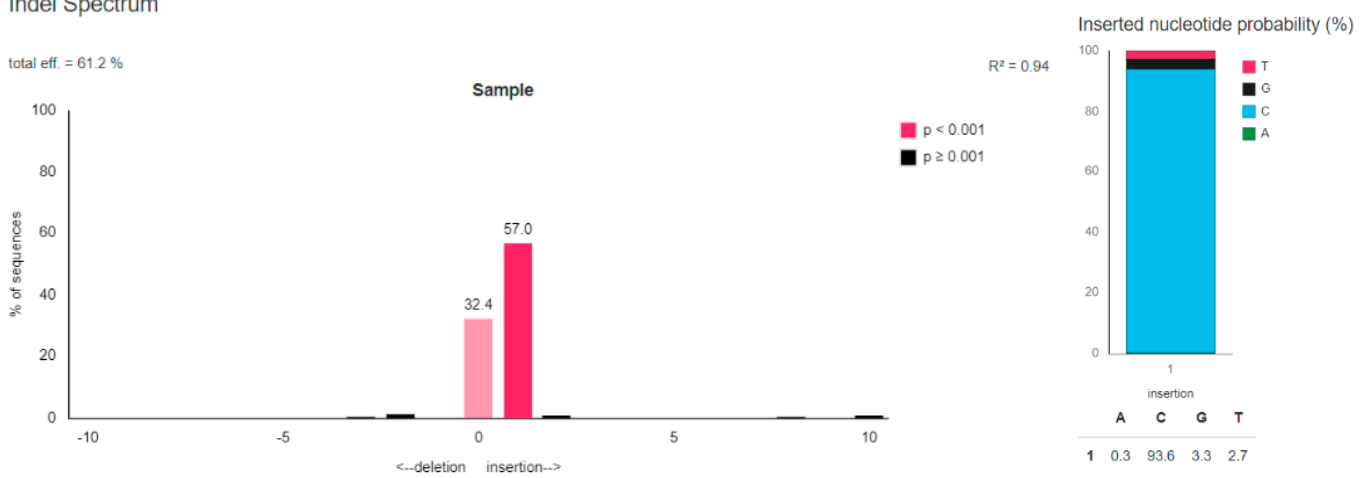

(b) gCMAHF3/R3

Indel Spectrum
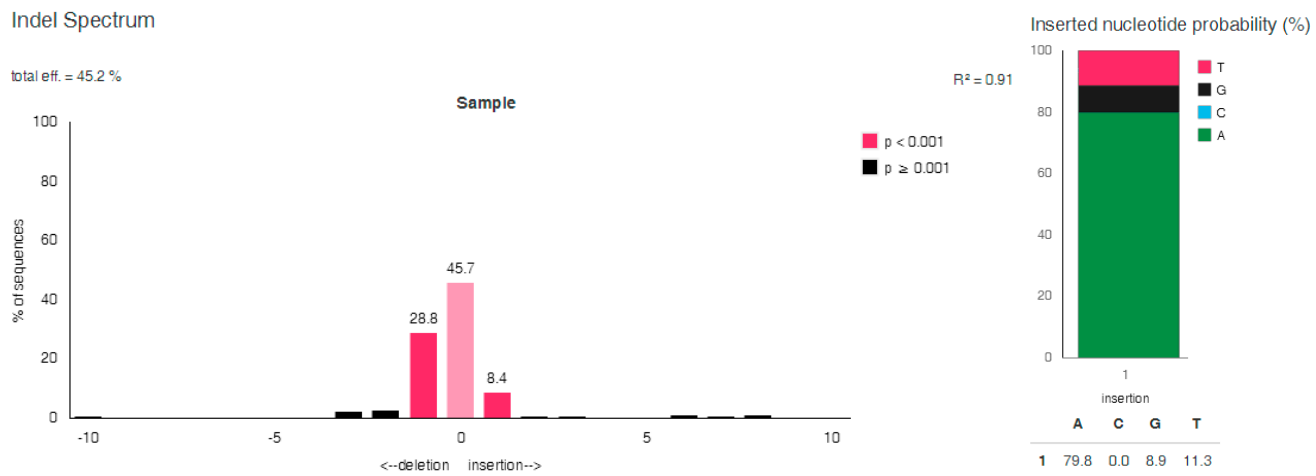

(c) g 34 GalNT2 F3/R3

Figure 2. Cont. 


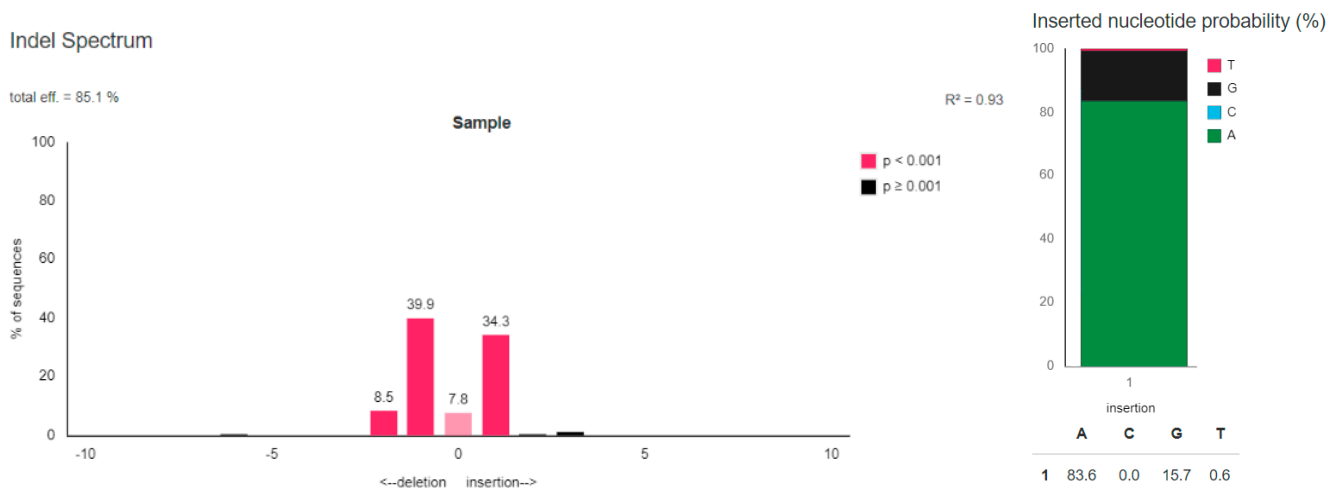

(d) gvWF F2/R2

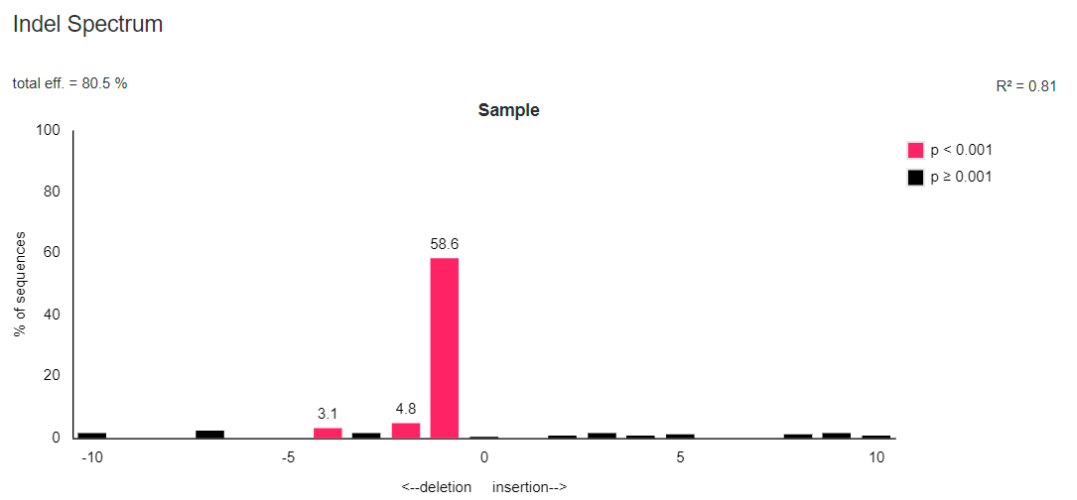

(e) gASGR1 F3/R3

Figure 2. The indel spectrum and inserted nucleotide probability results for the CRISPR/Cas9 genetic constructs containing gRNA chosen as the best for disruption of tested porcine genes. Results obtained after use the plasmids with (a) gGGTA1 F1/R1; (b) gCMAH F3/R3; (c) gß4GalNT2 F3/R3; (d) gvWF F2/R2; (e) gASGR1 F3/R3.

The indel spectrum and inserted nucleotide probability results, alignment, and decomposition quality controls for the other CRISPR/Cas9 genetic constructs containing gRNA tested for disruption of the porcine genes are present in the Supplement (Figure S1). The alignment and decomposition quality controls for the CRISPR/Cas9 genetic constructs containing gRNA chosen as the best for the disruption of the porcine genes are present in the Supplement (Figure S2).

3.2.3. Comparison of Bioinformatically Predicted DNA Diruption Efficiency Results with Those Obtained in the In Vitro Cultured Cells

The total efficiency results of modifications obtained using the CRISPR/Cas9 system predicted during bioinformatics analysis in silico were compared with those obtained in in vitro cultured porcine primary kidney fibroblasts. Comparison of the results for all tested construction variants is shown in Table 2.

Table 2. Comparison of the results of total efficiency predicted in silico with the results obtained in the in vitro cultured cells.

\begin{tabular}{ccc}
\hline gRNA & In Silico Analysis Predicted Total Efficiency & In Vitro Analysis Total Efficiency \\
\hline gGGTA1 F1/R1 & $78.9 \%$ & $70.1 \%$ * \\
gGGTA1 F2/R2 & $72.2 \%$ & $27.1 \%$ \\
gGGTA1 F3/R3 & $70.6 \%$ & $18.2 \%$ \\
gCMAH F1/R1 & $61.5 \%$ & $7.8 \%$ \\
\hline
\end{tabular}


Table 2. Cont.

\begin{tabular}{ccc}
\hline gRNA & In Silico Analysis Predicted Total Efficiency & In Vitro Analysis Total Efficiency \\
\hline gCMAH F2/R2 & $73.5 \%$ & $12.9 \%$ \\
gCMAH F3/R3 & $70.1 \%$ & $61.2 \%$ * \\
g $\beta 4$ GalNT2 F1/R1 & $53.3 \%$ & $8.1 \%$ \\
g 34 GalNT2 F2/R2 & $66 \%$ & $17.6 \%$ \\
g 34 GalNT2 F3/R3 & $75.8 \%$ & $45.2 \% *$ \\
gvWF F1/R1 & $48.9 \%$ & $5.2 \%$ \\
gvWF F2/R2 & $73.9 \%$ & $85.1 \% *$ \\
gvWF F3/R3 & $76 \%$ & $39.6 \%$ \\
gASGR1 F1/R1 & $72.7 \%$ & $13.9 \%$ \\
gASGR1 F2/R2 & $67.3 \%$ & $33.5 \%$ \\
gASGR1 F3/R3 & $68.7 \%$ & $80.5 \%$ * \\
\hline
\end{tabular}

* the gRNA together with total efficiency was marked, which was chosen as the best for disruption of the studied genes.

\subsection{Analysis of the Potential Off-Target Sites in Porcine Genome}

\subsubsection{Selection of the Potential Off-Target Sites Location}

After selecting the best genetic constructs containing gRNAs that mediate in the efficient disruption of studied porcine loci, off-target sites were predicted. For each targeted modification site, a minimum of three loci have been selected that can lead to mutations outside the target locus using the Benchling internet platform. The main criterion considered when choosing the tested off-target sites was the score showing the probability of a DNA break at a given off-target site by the testes construct is equal to or higher than 1.0. In addition, all off-target loci predicted in the coding sequences for selected constructs were checked. The list of tested potential off-target sites is presented in Table 3.

Table 3. A summary of bioinformatically predicted potential off-target sites for selected genetic constructs.

\begin{tabular}{|c|c|c|c|}
\hline Chosen gRNA & * & Sequence & Porcine Genome Localization \\
\hline \multirow{4}{*}{ gGGTA1 F1/R1 } & 1. & GCTGCACTTGAAGACCATCG & chr7: +33592796 \\
\hline & 2. & GATAGTCATGGAGACCATCG & $\begin{array}{c}\text { chr7: }+1925303 \\
\text { (RIPK1 gene: ENSSSCG00000001009) }\end{array}$ \\
\hline & 3. & CCTGCGCGTGAAGACCAACG & $\begin{array}{c}\text { chr2: }-44588168 \\
\text { (OTOG gene: ENSSSCG00000013376) }\end{array}$ \\
\hline & 4. & GAGGTGCATGAAGAACATCT & chr2: +13174290 \\
\hline \multirow{3}{*}{ gCMAH F3/R3 } & 5. & ATTCGATCCTCCТАACСССТ & chr15: +40937188 \\
\hline & 6. & TCTTAACCCTCATAACCCGT & chr4: -97128319 \\
\hline & 7. & AATAAATCACCCTAACCAGT & $\begin{array}{c}\text { chr4: }+116709576 \\
\text { (HIPK1 gene: ENSSSCG00000006760) }\end{array}$ \\
\hline \multirow{3}{*}{ g $\beta 4$ Gal NT2 F3/R3 } & 8. & AAACTACCAGCTCCACAGAG & chr16: -5677136 \\
\hline & 9. & ATTGTACCACCTCCACAGAC & chr10: -13115375 \\
\hline & 10. & TCAGTATCACCTCCACAGAG & chr7: -109695811 \\
\hline \multirow{4}{*}{ gvWF F2/R2 } & 11. & ССTTCTGCTTCATGCCCGCG & chr6: +157052374 \\
\hline & 12. & GCACGTACTCCTTGCCCGCG & $\begin{array}{c}\text { chr4: }-347951 \\
\text { (ARHGAP39 gene: ENSSSCG00000005894) }\end{array}$ \\
\hline & 13. & CCGTGTCGTCCAGGCCCGCG & $\begin{array}{c}\text { chr6: -9684887 } \\
\text { (WWOX gene: ENSSSCG00000027415) }\end{array}$ \\
\hline & 14. & СССТGTCCTGCAGGCCTGCG & $\begin{array}{c}\text { chr14: }-55036169 \\
\text { (COMT gene: ENSSSCG00000010132) }\end{array}$ \\
\hline
\end{tabular}


Table 3. Cont.

\begin{tabular}{cccc}
\hline Chosen gRNA & $*$ & \multicolumn{1}{c}{ Sequence } & Porcine Genome Localization \\
\hline & 15. & GCATATGTCTGGTACGGGCA & chr6: +4310372 \\
\cline { 2 - 4 } & 16. & GCATATGTCTGGTACGGGCA & chr6: -4126764 \\
\cline { 2 - 4 } gASGR1 F3/R3 & 17. & CACAATGACAGGTACGGGCA & $\begin{array}{c}\text { chr5: }+67768944 \\
(\text { KCNA1 gene: ENSSSCG00000000716) }\end{array}$ \\
\cline { 2 - 4 } & 18. & CCAGACGACTGGCACGGGCA & chr12: -54751930 \\
\cline { 2 - 4 } & 19. & CCCGCTGTCTGGGACGGGCA & $\begin{array}{c}\text { chr6: }+155398812 \\
\text { (C1orf210 gene: ENSSSCG00000003951) }\end{array}$ \\
\hline
\end{tabular}

Nucleotides not complementary to the target template for a given gRNA were determined with the bold font. * The number of the selected off-target site.

\subsubsection{TIDE Analysis of the Chosen Potential Off-Target Sites}

The presence of unwanted changes-the indel mutations, in eight off-target sites from the 19 tested loci was confirmed using the TIDE tool. The off-target sites were confirmed in loci numbers 1, 4, 5, $10,11,14,18$, and 19. Only in one locus the occurred modification was statistically significant at the level $p<0.001$ and it was site number 1 . It was determined that the off-target site number 1 for the genetic construct of gGGTA1 F1/R1 was cut with a total efficiency of $3.9 \%$. A mutation arising with the statistical significance was the insertion of one nucleotide, which occurred in $1.7 \%$ sequences. Guanosine nucleotide was inserted in the $50.8 \%$ sequences. The indel spectrum and inserted nucleotide probability for the number 1 off-target locus is presented in Figure 3.
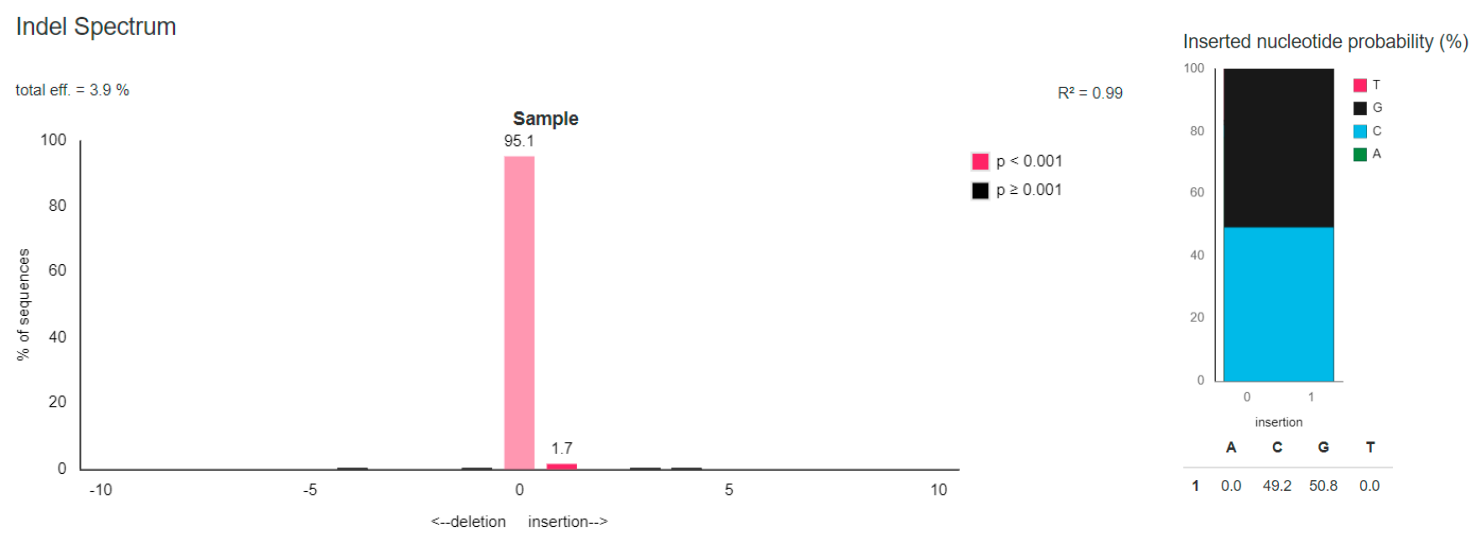

Figure 3. The indel spectrum and inserted nucleotide probability result for the number 1 off-target locus after the use of CRISPR/Cas9 genetic construct containing gGGTA1 F1/R1 chosen as the best for disruption of porcine GGTA1 gene.

3.3.3. Comparison of Bioinformatically Predicted DNA Diruption Efficiency Results in the Off-Target Sites with Those Obtained in the In Vitro Cultured Cells

The efficiency results of off-target mutation using the CRISPR/Cas9 system obtained during bioinformatics analysis were compared with those obtained in in vitro cultures. The results are summarized in Table 4. 
Table 4. Comparison of bioinformatic analysis with the results of laboratory analyses-analysis of the off-target sites presence.

\begin{tabular}{|c|c|c|c|}
\hline \multirow{2}{*}{ gRNA } & \multirow{2}{*}{ * } & \multicolumn{2}{|c|}{ DNA Hydrolysis Efficiency at a Potential Off-Target Site } \\
\hline & & Bioinformatic Analysis & In Vitro Cultured Cells \\
\hline \multirow{4}{*}{ gGGTA1 F1/R1 } & 1. & $1.6 \%$ & $3.9 \%$ \\
\hline & 2. & $0.5 \%$ & $0 \%$ \\
\hline & 3. & $0.4 \%$ & $0 \%$ \\
\hline & 4. & $0.2 \%$ & $0.8 \%$ \\
\hline \multirow{3}{*}{ gCMAH F3/R3 } & 5. & $0.6 \%$ & $1.1 \%$ \\
\hline & 6. & $0.6 \%$ & $0 \%$ \\
\hline & 7. & $0.2 \%$ & $0 \%$ \\
\hline \multirow{3}{*}{ gB4GalNT2 F3/R3 } & 8. & $2.6 \%$ & $0 \%$ \\
\hline & 9. & $1.8 \%$ & $0 \%$ \\
\hline & 10. & $1.8 \%$ & $2.2 \%$ \\
\hline \multirow{4}{*}{ gvWF F2/R2 } & 11. & $0.9 \%$ & $1.8 \%$ \\
\hline & 12. & $0.8 \%$ & $0 \%$ \\
\hline & 13. & $0.4 \%$ & $0 \%$ \\
\hline & 14. & $0.2 \%$ & $3.4 \%$ \\
\hline \multirow{5}{*}{ gASGR1 F3/R3 } & 15. & $1.5 \%$ & $0 \%$ \\
\hline & 16. & $1.5 \%$ & $0 \%$ \\
\hline & 17. & $1.4 \%$ & $0 \%$ \\
\hline & 18. & $0.7 \%$ & $1.7 \%$ \\
\hline & 19. & $0.6 \%$ & $1.6 \%$ \\
\hline
\end{tabular}

* The number of the selected off-target site.

\section{Discussion}

The research presented in this paper shows the importance of the verification of the bioinformatically selected gRNAs in the context of the modifications obtaining efficiency. To compare the efficiency of individual gRNAs in modifications obtaining in cells cultured in vitro, it was necessary to use the third-generation CRISPR/Cas9 system, which enables to carry out antibiotic selection using puromycin after transfection. This process eliminates the impact of the transfection efficiency associated with the chosen method on the efficiency of obtaining modifications via individual gRNAs [28-30]. After antibiotic selection, a population of positively transfected cells after nucleofection was examined. In this way, it was possible to determine the efficiency of indel mutation formation at target sites for specific gRNAs. It was shown that the results obtained in the cells prepared in this way slightly coincided with bioinformatics predictions.

Therefore, the selection of gRNA itself, which would mediate the disruption of specific genomic site should be given the great importance. There are many different online tools that enable gRNA design. The limiting factor are the available databases related to the genomes of various organisms. Thus, the selection of the appropriate tool for prediction of Cas9 DNA hydrolysis target sites is based on the chosen research model [31,32].

The results of sequencing of PCR products that were amplified within the target sites of designed gRNAs were analyzed using the TIDE tool. This method makes it easy to determine, based on the results of sequencing, the indel mutation profile that occurs in a population of genetically modified cells using the CRISPR/Cas9 system. The indel detection by amplicon analysis (IDAA) method is also used for this purpose. It is based on three-primer amplicon labeling and detection by capillary 
electrophoresis [33]. The sensitivity and precision of both methods to determine the insertion-deletion profile of the modification is similar. The choice of method used depends on the researcher's preferences and available infrastructure [34,35].

Based on the results obtained in this study, the best targeted sites were selected within the genes tested, thanks to which it is possible to obtain modifications efficiently. The assessment of individual gRNAs is very important in the context of obtaining simultaneous multimodification of the porcine genome for xenotransplantation purposes. This is the latest goal of researchers in this field. Research related to this work is part of this trend. Modifications used in the research are aimed at counteracting the two immune barriers existing in xenotransplantation. The knock-out of the porcine GGTA1, CMAH and $\beta 4$ GalNT2 genes aims at the elimination of the hyperacute xenograft rejection. Prevention of the coagulation dysregulation can be achieved by the disruption of the porcine $v W F$ gene for lung xenotransplantation and knock-out of the porcine ASGR1 gene for liver xenotransplantation.

An important aspect related to these results is the ethical effect. Preliminary evaluation of bioinformatic data allows to limit the participation of animals in research. This is in line with the Polish recommendations of the National Ethics Committee for Animal Experiments described in Resolution No. 14/2017, as well as with international guidelines and trends.

This study also analyzes the off-target sites generated by Cas9 nuclease in combination with the best gRNAs selected. The formation of additional DNA hydrolysis sites by Cas9 is a common problem observed in CRISPR/Cas9-related studies. Off-target mutations are the biggest threat associated with the use of the new genome editing technology. In total, 19 potential off-target sites were tested for genetic constructs selected during experiments (Figure 3, Figures S3-S7). Off-target mutations were confirmed at eight loci. Only in one of them the changes occurred with statistical significance at the level of $p<0.001$. This was site No. 1 (for genetic construction containing gGGTA1 F1/R1). Eight potential off-target sites were in the coding sequences of the porcine genome. Experimentally, in genetically modified porcine primary kidney fibroblast cultures, the presence of the off-target mutations in two gene coding sequences was confirmed. The first locus in which off-target mutation has been reviled after the use of the genetic construction with gvWF F2/R2 lies within the porcine COMT gene. Another one confirmed off-target site for gASGR1 F3/R3 containing constructs is within the porcine C1orf210 gene. These loci must be checked after receiving genetically modified animals using obtained using these two genetic constructs.

The obtained results correlate with the research that describe the influence of the non-complementary nucleotides (mismatch nucleotides) position between gRNA and the sequence of the potential off-target site. The efficiency of the off-target mutation formation is higher when incomplementarity occurs in the eighth nucleotide and in the first three nucleotides of the gRNA sequence $\left(5^{\prime} \rightarrow 3^{\prime}\right)$. For nucleotide in the eighth position $\left(5^{\prime} \rightarrow 3^{\prime}\right)$ such a relationship was confirmed for two out of eight off-target sites (numbers 1 and 19) for the examined gRNAs in this study. In addition, as many as seven out of eight off-target sites (numbers 1,4,5,10,11,18, and 19) had a mismatch of at least one nucleotide in the first, second, or third position of the gRNA sequence $\left(5^{\prime} \rightarrow 3^{\prime}\right)$ [36]. Interestingly, this study also revealed that in three out of eight confirmed off-target sites, complementarity was found for the seventh position nucleotide. Studies show that the CRISPR/Cas9 system tolerates three to five mismatches in the distal gRNA region from the protospacer adjacent motif (PAM) sequence. In addition, it has been reported that the complementary alignment of the ten nucleotides of the proximal gRNA region from the PAM sequence is sufficient to mediate the Cas9 nuclease DNA hydrolysis [37]. It has also been shown that the occurrence of nucleotide incomplementarity at positions 15, 16, and 17 of the gRNA sequence $\left(5^{\prime} \rightarrow 3^{\prime}\right)$ to a potential off-target site abolishes CRISPR/Cas9 system activity [38].

The research presented above proved the usefulness of the TIDE online tool for off-target sites checking. There are other, more accurate methods used for this purpose. One of them is DISCOVER-Seq (discovery of in situ Cas off-targets and verification by sequencing), which uses recruitment of the factors involved in DNA repair. To this end, the binding of double-strand break DNA repair protein (MRE11) is tracked. Thanks to this it is possible to check the double-strand DNA breaks in the whole 
genome [39]. Another method is CIRCLE-seq (circularization for in vitro reporting of cleavage effects by sequencing), it consists of next generation sequencing and leads to the analysis of the presence of off-target sites throughout the genome [40]. Both above methods have many advantages, so it would be worth to additionally perform these analyzes for the best genetic constructions selected in this paper. However, their use is only possible in in vitro cell culture studies and it is not possible to evaluate off-target sites in genetically modified animals. In contrast, the TIDE tool enables quick and cheap recognition of basic off-target sites, and its use can apply to both cellular and model animals research.

New approaches have emerged to reduce the risk of off-target mutations after using the CRISPR/Cas9 system. One of them is the use of new algorithms in bioinformatics tools, thanks to which it is possible to more accurately assess the efficiency of modifications within individual off-target sites [41-44]. Another approach is to use modified Cas9 with nickase activity. It has been proven that introducing modifications with the CRISPR/Cas9 system modified in such a way reduces the number of unwanted mutations $[45,46]$. The second approach is to provide Cas9 protein and gRNA in the form of a ribonucleoprotein complex (RNP). It has been shown that this method of delivery of the CRISPR/Cas9 system enabled obtaining the modifications at the target site with high efficiency. The number of the off-target mutations that have arisen has significantly decreased. However, the use of the RNP complex has a major drawback. There is a problem with the number of complexes delivered. Very high concentrations are used because only such concentrations can guarantee that some of them will function in the cell nuclei of genetically modified cell cultures in vitro. High concentration of the RNP complexes may have a cytotoxic effect on some cell lines [47-51]. Accordingly, ribonucleoprotein complexes can only be used in some experiments.

\section{Conclusions}

The research presented above enabled the usefulness assessment of the CRISPR/Cas9 system genetic constructions containing gRNAs to obtain modifications for xenotransplantation purposes. Selected gRNAs, which in combination with Cas9 nuclease enable for the efficient disruption of the studied genes, can be used to obtain genetically modified pigs. To obtain an effect associated with counteracting the immune response in the recipient — the primate animal models or human, all the modifications tested should be present in the porcine genome simultaneously. The genetically modified porcine primary kidney fibroblasts cells nuclei for the SCNT procedure or verified genetic constructs for microinjection of the porcine zygotes can be used to obtain the desired porcine genome modifications. After receiving genetically modified animals, it is necessary to check off-target sites, which presence has been confirmed by the above analyzes.

Supplementary Materials: The indel spectrum results, insertion nucleotides probability results, alignment and decomposition quality controls for studied genetic constructs are available online at http://www.mdpi.com/ 2073-4425/11/6/713/s1, Figure S1: The indel spectrum and inserted nucleotide probability results, alignment, and decomposition quality controls for the other CRISPR/Cas9 genetic constructs containing gRNA tested for disruption of the porcine genes.; Figure S2: The alignment and decomposition quality controls for the CRISPR/Cas9 genetic constructs containing gRNA chosen as the best for the disruption of the porcine genes; Figure S3: The indel spectrum for the numbers 2-4 off-target loci after the use of CRISPR/Cas9 genetic construct containing gGGTA1 F1/R1 chosen as the best for disruption of porcine GGTA1 gene; Figure S4: The indel spectrum for the numbers 5-7 off-target loci after the use of CRISPR/Cas9 genetic construct containing gCMAH F3/R3 chosen as the best for disruption of porcine CMAH gene; Figure S5: The indel spectrum for the number 8-10 off-target loci after the use of CRISPR/Cas9 genetic construct containing g $\beta 4$ GalNT2 F3/R3 chosen as the best for disruption of porcine $\beta 4$ GalNT2 gene; Figure S6: The indel spectrum for the numbers 11-14 off-target loci after the use of CRISPR/Cas9 genetic construct containing gvWF F2/R2 chosen as the best for disruption of porcine $v W F$ gene; Figure S7: The indel spectrum for the numbers 15-19 off-target loci after the use of CRISPR/Cas9 genetic construct containing gASGR1 F3/R3 chosen as the best for disruption of porcine ASGR1 gene.

Author Contributions: Investigation and writing-original draft, review and editing, N.R.; supervision and conceptualization, M.H., D.L.; supervision, J.Z.; funding acquisition and supervision, R.S. All authors have read and agreed to the published version of the manuscript. 
Funding: This research was partially funded by the National Centre for Research and Development (Grant No. INNOMED/I/17/NCBR/2014) within the framework of the INNOMED programme entitled "Development of an Innovative Technology Using Transgenic Porcine Tissues for Bio-Medical Purposes," acronym: MEDPIG. The publication was co-financed within the framework of a Ministry of Science and Higher Education program as "Regional Initiative Excellence" in the years 2019-2022, Project No. 005/RID/2018/19.

Conflicts of Interest: The authors declare no conflict of interest.

\section{References}

1. Ekser, B.; Li, P.; Cooper, D.K.C. Xenotransplantation: Past, present, and future. Curr. Opin. Organ. Transplant. 2017, 22, 513-521. [CrossRef] [PubMed]

2. Cooper, D.K.C.; Ekser, B.; Tector, J. A brief history of clinical xenotransplantation. Int. J. Surg. 2015, 23, 205-210. [CrossRef] [PubMed]

3. Aristizabal, A.M.; Caicedo, L.A.; Martínez, J.M.; Moreno, M.; Echeverri, G.J. Clinical xenotransplantation, a closer reality: Literature review. Cirugía Española (English Ed.) 2017, 95, 62-72. [CrossRef]

4. Cooper, D.K.C.; Iwase, H.; Wang, L.; Yamamoto, T.; Li, Q.; Li, J.; Zhou, H.; Hara, H. Bringing home the bacon: Update on the state of kidney xenotransplantation. Blood Purif. 2018, 45, 254-259. [CrossRef] [PubMed]

5. Vadori, M.; Cozzi, E. The immunological barriers to xenotransplantation. Tissue Antigens 2015, 86, $239-253$. [CrossRef] [PubMed]

6. Cooper, D.K.C.; Ekser, B.; Tector, J. Immunobiological barriers to xenotransplantation. Int. J. Surg. 2015, 23, 211-216. [CrossRef]

7. Fischer, K.; Rieblinger, B.; Hein, R.; Sfriso, R.; Zuber, J.; Fischer, A.; Klinger, B.; Liang, W.; Flisikowski, K.; Kurome, M.; et al. Viable pigs after simultaneous inactivation of porcine MHC class I and three xenoreactive antigen genes GGTA1, CMAH and B4GALNT2. Xenotransplantation 2020, 27, e12560. [CrossRef]

8. Ganji, M.-R.; Broumand, B. Acute cellular rejection. Iran. J. Kidney Dis. 2007, 1, 54-56.

9. Lin, C.C.; Cooper, D.K.C.; Dorling, A. Coagulation dysregulation as a barrier to xenotransplantation in the primate. Transpl. Immunol. 2009, 21, 75-80. [CrossRef]

10. Cooper, D.K.C.; Ezzelarab, M.B.; Hara, H.; Iwase, H.; Lee, W.; Wijkstrom, M.; Bottino, R. The pathobiology of pig-to-primate xenotransplantation: A historical review. Xenotransplantation 2013, 23, 83-105. [CrossRef]

11. Chuang, C.K.; Chen, C.H.; Huang, C.L.; Su, Y.H.; Peng, S.H.; Lin, T.Y.; Tai, H.C.; Yang, T.S.; Tu, C.F. Generation of GGTA1 mutant pigs by direct pronuclear microinjection of CRISPR/Cas9 plasmid vectors. Anim. Biotechnol. 2017, 28, 174-181. [CrossRef] [PubMed]

12. Lin, S.S.; Hanaway, M.J.; Gonzalez-Stawinski, G.V.; Lau, C.L.; Parker, W.; Davis, R.D.; Byrne, G.W.; Diamond, L.E.; Logan, J.S.; Platt, J.L. The role of anti-Galalpha1-3Gal antibodies in acute vascular rejection and accommodation of xenografts. Transplantation 2000, 70, 1667-1674. [CrossRef] [PubMed]

13. Scobie, L.; Padler-Karavani, V.; Le Bas-Bernardet, S.; Crossan, C.; Blaha, J.; Matouskova, M.; Hector, R.D.; Cozzi, E.; Vanhove, B.; Charreau, B.; et al. Long-term IgG response to porcine Neu5Gc antigens without transmission of PERV in burn patients treated with porcine skin xenografts. J. Immunol. 2013, 191, 2907-2915. [CrossRef] [PubMed]

14. Varki, A. Loss of N-glycolylneuraminic acid in humans: Mechanisms, consequences, and implications for hominid evolution. Am. J. Phys. Anthropol. 2001, 116, 54-69. [CrossRef] [PubMed]

15. Byrne, G.; Ahmad-Villiers, S.; Du, Z.; McGregor, C. B4GalNT2 and xenotransplantation: A newly appreciated xenogeneic antigen. Xenotransplantation 2018, 25, e12394. [CrossRef] [PubMed]

16. Byrne, G.W.; Du, Z.; Stalboerger, P.; Kogelberg, H.; McGregor, C.G.A. Cloning and expression of porcine $\beta 1,4 \mathrm{~N}$-acetylgalactosaminyl transferase encoding a new xenoreactive antigen. Xenotransplantation 2014, 21, 543-554. [CrossRef] [PubMed]

17. Wang, Z.Y.; Burlak, C.; Estrada, J.L.; Li, P.; Tector, M.F.; Tector, A.J. Erythrocytes from GGTA1/CMAH knockout pigs: Implications for xenotransfusion and testing in non-human primates. Xenotransplantation 2014, 21, 376-384. [CrossRef] [PubMed]

18. Cooper, D.K.C.; Bottino, R. Recent advances in understanding xenotransplantation: Implications for the clinic. Expert Rev. Clin. Immunol. 2015, 11, 1379-1390. [CrossRef]

19. Bühler, L.; Basker, M.; Alwayn, I.P.; Goepfert, C.; Kitamura, H.; Kawai, T.; Gojo, S.; Kozlowski, T.; Ierino, F.L.; Awwad, M.; et al. Coagulation and thrombotic disorders associated with pig organ and hematopoietic cell transplantation in nonhuman primates. Transplantation 2000, 70, 1323-1331. [CrossRef] 
20. Lau, C.L.; Cantu, E.; Gonzalez-Stawinski, G.V.; Holzknecht, Z.E.; Nichols, T.C.; Posther, K.E.; Rayborn, C.A.; Platt, J.L.; Parker, W.; Davis, R.D. The role of antibodies and von Willebrand factor in discordant pulmonary xenotransplantation. Am. J. Transplant. 2003, 3, 1065-1075. [CrossRef]

21. Cantu, E.; Balsara, K.R.; Li, B.; Lau, C.; Gibson, S.; Wyse, A.; Baig, K.; Gaca, J.; Gonzalez-Stawinski, G.V.; Nichols, T.; et al. Prolonged function of macrophage, von Willebrand factor-deficient porcine pulmonary xenografts. Am. J. Transplant. 2007, 7, 66-75. [CrossRef] [PubMed]

22. Paris, L.L.; Estrada, J.L.; Li, P.; Blankenship, R.L.; Sidner, R.A.; Reyes, L.M.; Montgomery, J.B.; Burlak, C.; Butler, J.R.; Downey, S.M.; et al. Reduced human platelet uptake by pig livers deficient in the asialoglycoprotein receptor 1 protein. Xenotransplantation 2015, 22, 203-210. [CrossRef] [PubMed]

23. Li, P.; Estrada, J.L.; Burlak, C.; Montgomery, J.; Butler, J.R.; Santos, R.M.; Wang, Z.Y.; Paris, L.L.; Blankenship, R.L.; Downey, S.M.; et al. Efficient generation of genetically distinct pigs in a single pregnancy using multiplexed single-guide RNA and carbohydrate selection. Xenotransplantation 2015, 22, $20-31$. [CrossRef] [PubMed]

24. Jinek, M.; Chylinski, K.; Fonfara, I.; Hauer, M.; Doudna, J.A.; Charpentier, E. A programmable dual-RNA-guided DNA endonuclease in adaptive bacterial immunity. Science 2012, 337, 816-821. [CrossRef]

25. Du, J.; Yin, N.; Xie, T.; Zheng, Y.; Xia, N.; Shang, J.; Chen, F.; Zhang, H.; Yu, J.; Liu, F. Quantitative assessment of HR and NHEJ activities via CRISPR/Cas9-induced oligodeoxynucleotide-mediated DSB repair. DNA Repair 2018, 70, 67-71. [CrossRef]

26. Miyaoka, Y.; Mayerl, S.J.; Chan, A.H.; Conklin, B.R. Detection and quantification of HDR and NHEJ induced by genome editing at endogenous gene loci using droplet digital PCR. Methods Mol. Biol. 2018, 1768, 349-362. [CrossRef]

27. Fu, Y.; Foden, J.A.; Khayter, C.; Maeder, M.L.; Reyon, D.; Joung, J.K.; Sander, J.D. High-frequency off-target mutagenesis induced by CRISPR-Cas nucleases in human cells. Nat. Biotechnol. 2013, 31, 822-826. [CrossRef]

28. Lentsch, E.; Li, L.; Pfeffer, S.; Ekici, A.B.; Taher, L.; Pilarsky, C.; Grützmann, R. CRISPR/Cas9-mediated knock-out of krasG12D mutated pancreatic cancer cell lines. Int. J. Mol. Sci. 2019, 20, 5706. [CrossRef]

29. Yang, H.; Wang, J.; Zhao, M.; Zhu, J.; Zhang, M.; Wang, Z.; Gao, Y.; Zhu, W.; Lu, H. Feasible development of stable HEK293 clones by CRISPR/Cas9-mediated site-specific integration for biopharmaceuticals production. Biotechnol. Lett. 2019, 41, 941-950. [CrossRef]

30. Steyer, B.; Bu, Q.; Cory, E.; Jiang, K.; Duong, S.; Sinha, D.; Steltzer, S.; Gamm, D.; Chang, Q.; Saha, K. Scarless Genome Editing of Human Pluripotent Stem Cells via Transient Puromycin Selection. Stem Cell Rep. 2018, 10, 642-654. [CrossRef]

31. Doench, J.G.; Fusi, N.; Sullender, M.; Hegde, M.; Vaimberg, E.W.; Donovan, K.F.; Smith, I.; Tothova, Z.; Wilen, C.; Orchard, R.; et al. Optimized sgRNA design to maximize activity and minimize off-target effects of CRISPR-Cas9. Nat. Biotechnol. 2016, 34, 184-191. [CrossRef]

32. Cui, Y.; Xu, J.; Cheng, M.; Liao, X.; Peng, S. Review of CRISPR/Cas9 sgRNA Design Tools. Interdiscip. Sci. 2018, 10, 455-465. [CrossRef] [PubMed]

33. Yang, Z.; Steentoft, C.; Hauge, C.; Hansen, L.; Thomsen, A.L.; Niola, F.; Vester-Christensen, M.B.; Frödin, M.; Clausen, H.; Wandall, H.H.; et al. Fast and sensitive detection of indels induced by precise gene targeting. Nucleic Acids Res. 2015, 43, e59. [CrossRef] [PubMed]

34. Sentmanat, M.F.; Peters, S.T.; Florian, C.P.; Connelly, J.P.; Pruett-Miller, S.M. A survey of validation strategies for CRISPR-Cas9 editing. Sci. Rep. 2018, 8, 888. [CrossRef] [PubMed]

35. Kosicki, M.; Rajan, S.S.; Lorenzetti, F.C.; Wandall, H.H.; Narimatsu, Y.; Metzakopian, E.; Bennett, E.P. Dynamics of indel profiles induced by various CRISPR/Cas9 delivery methods. Prog. Mol. Biol. Transl. Sci. 2017, 152, 49-67. [CrossRef]

36. Peng, H.; Zheng, Y.; Zhao, Z.; Liu, T.; Li, J. Recognition of CRISPR/Cas9 off-target sites through ensemble learning of uneven mismatch distributions. Bioinformatics 2018, 34, i757-i765. [CrossRef]

37. Kuscu, C.; Arslan, S.; Singh, R.; Thorpe, J.; Adli, M. Genome-wide analysis reveals characteristics of off-target sites bound by the Cas9 endonuclease. Nat. Biotechnol. 2014, 32, 677-683. [CrossRef]

38. Zheng, T.; Hou, Y.; Zhang, P.; Zhang, Z.; Xu, Y.; Zhang, L.; Niu, L.; Yang, Y.; Liang, D.; Yi, F.; et al. Profiling single-guide RNA specificity reveals a mismatch sensitive core sequence. Sci. Rep. 2017, 7, 40638. [CrossRef]

39. Wienert, B.; Wyman, S.K.; Richardson, C.D.; Yeh, C.D.; Akcakaya, P.; Porritt, M.J.; Morlock, M.; Vu, J.T.; Kazane, K.R.; Watry, H.L.; et al. Unbiased detection of CRISPR off-targets in vivo using DISCOVER-Seq. Science 2019, 364, 286-289. [CrossRef] 
40. Tsai, S.Q.; Nguyen, N.T.; Malagon-Lopez, J.; Topkar, V.V.; Aryee, M.J.; Joung, J.K. CIRCLE-seq: A highly sensitive in vitro screen for genome-wide CRISPR-Cas9 nuclease off-targets. Nat. Methods 2017, 14, 607-614. [CrossRef]

41. Pliatsika, V.; Rigoutsos, I. Off-Spotter': Very fast and exhaustive enumeration of genomic lookalikes for designing CRISPR/Cas guide RNAs. Biol. Direct 2015, 10, 4. [CrossRef] [PubMed]

42. Xiao, A.; Cheng, Z.; Kong, L.; Zhu, Z.; Lin, S.; Gao, G.; Zhang, B. CasOT: A genome-wide Cas9/gRNA off-target searching tool. Bioinformatics 2014, 30, 1180-1182. [CrossRef] [PubMed]

43. Xiong, Y.; Xie, X.; Wang, Y.; Ma, W.; Liang, P.; Songyang, Z.; Dai, Z. pgRNAFinder: A web-based tool to design distance independent paired-gRNA. Bioinformatics 2017, 33, 3642-3644. [CrossRef] [PubMed]

44. Zhu, L.J.; Holmes, B.R.; Aronin, N.; Brodsky, M.H. CRISPRseek: A Bioconductor package to identify target-specific guide RNAs for CRISPR-Cas9 genome-editing systems. PLoS ONE 2014, 9, e108424. [CrossRef]

45. Cong, L.; Ran, F.A.; Cox, D.; Lin, S.; Barretto, R.; Habib, N.; Hsu, P.D.; Wu, X.; Jiang, W.; Marraffini, L.A.; et al. Multiplex Genome Engineering Using CRISPR/Cas Systems. Science 2013, 339, 819-823. [CrossRef]

46. Fu, B.; Smith, J.D.; Fuchs, R.T.; Mabuchi, M.; Curcuru, J.; Robb, G.B.; Fire, A.Z. Target-dependent nickase activities of the CRISPR-Cas nucleases Cpf1 and Cas9. Nat. Microbiol. 2019, 4, 888-897. [CrossRef]

47. Lyu, P.; Javidi-Parsijani, P.; Atala, A.; Lu, B. Delivering Cas9/sgRNA ribonucleoprotein (RNP) by lentiviral capsid-based bionanoparticles for efficient 'hit-and-run' genome editing. Nucleic Acids Res. 2019, 47, e99. [CrossRef]

48. Martin, R.M.; Ikeda, K.; Cromer, M.K.; Uchida, N.; Nishimura, T.; Romano, R.; Tong, A.J.; Lemgart, V.T.; Camarena, J.; Pavel-Dinu, M.; et al. Highly Efficient and Marker-free Genome Editing of Human Pluripotent Stem Cells by CRISPR-Cas9 RNP and AAV6 Donor-Mediated Homologous Recombination. Cell Stem Cell 2019, 24, 821-828.e5. [CrossRef]

49. Oh, S.A.; Seki, A.; Rutz, S. Ribonucleoprotein Transfection for CRISPR/Cas9-Mediated Gene Knockout in Primary T Cells. Curr. Protoc. Immunol. 2019, 124, e69. [CrossRef]

50. Seki, A.; Rutz, S. Optimized RNP transfection for highly efficient CRI SPR/Cas9-mediated gene knockout in primary T cells. J. Exp. Med. 2018, 215, 985-997. [CrossRef]

51. Vakulskas, C.A.; Behlke, M.A. Evaluation and reduction of crispr off-target cleavage events. Nucleic Acid Ther. 2019, 29, 167-174. [CrossRef] [PubMed]

(C) 2020 by the authors. Licensee MDPI, Basel, Switzerland. This article is an open access article distributed under the terms and conditions of the Creative Commons Attribution (CC BY) license (http://creativecommons.org/licenses/by/4.0/). 